\title{
Estudo do tempo de excreção renal pela cintilografia em felinos domésticos
}

\begin{abstract}
Georgea Bignardi JARRETTA ${ }^{1}$ Pedro Primo BOMBONATO ${ }^{2}$ Benedicto Wlademir DE MARTIN $^{3}$
\end{abstract}

\section{Correspondência para:}

Georgea Bignardi Jarretta, R. São Jose, 134, ap. 01, Embaré, Santos - SP, CEP. 11040-200

georgeabj@yahoo.com/

georgeabj@hotmail.com

Recebido para publicação: 02/07/2007 Aprovado para publicação: 29/10/2009

\author{
1- Universidade Metropolitana de Santos e Universidade Monte Serrat, \\ Santos-SP \\ 2- Departamento de Cirurgia da Faculdade de Medicina Veterinária e \\ Zootecnia da Universidade de São Paulo, São Paulo-SP \\ 3- Instituto Veterinário de Imagem, São Paulo-SP
}

\section{Resumo}

Palavras-chave: Medicina Nuclear. Cintilografia.

$\mathrm{Na}$ rotina clínica de felinos domésticos, algumas modalidades de diagnóstico por imagem, como a ultrassonografia, radiografia simples e urografia excretora, já são amplamente utilizadas. A cintilografia é uma técnica não invasiva, capaz de oferecer informaçóes funcionais de rins individualmente, porém é considerada uma modalidade menos usual. O objetivo deste estudo foi verificar o tempo de excreção renal de felinos domésticos por meio da cintilografia, em animais com parâmetros ultrassonográficos e radiográficos dentro dos limites da normalidade. Foram utilizados 15 animais, nove machos e seis fêmeas, e estes foram divididos em grupos de animais náo submetidos à anestesia e anestesiados. Foi estabelecido o tempo para o radiofármaco obter acúmulo máximo em cada um dos rins e o tempo para este acúmulo máximo ser reduzido à metade. Não houve diferença estatística entre os valores dos animais não-anestesiados e anestesiados, nem entre os rins esquerdo e direito, tampouco entre machos e fêmeas.

\section{Introdução}

Os felinos domésticos são acometidos por diversas doenças renais que podem levar à insuficiência funcional do órgão; todavia, a presença de doença renal não traduz necessariamente uma insuficiência renal, pois isso vai depender da quantidade do parênquima afetado e da gravidade das lesôes. Felinos domésticos também são comumente acometidos por doenças obstrutivas do trato urinário, como a doença do trato urinário inferior (DTUIF), podendo levar a um comprometimento da excreção renal ${ }^{1}$.

A avaliação funcional de rins de felinos domésticos é considerada uma importante informação na clínica desta espécie, para que, na presença de doença renal, esta possa ser detectada e tratada antes da instalação da insuficiência renal nestes animais. Testes laboratoriais como a mensuração sérica de ureia e creatinina podem avaliar a função renal; porém, possuem baixa sensibilidade, já que a azotemia renal está presente quando cerca de $75 \%$ dos néfrons estiver comprometido, não havendo especificidade com relação à função individual dos rins ${ }^{1}$. A detecção precoce das afecçóes renais agudas é importante para a implantação de um tratamento adequado que minimize os danos tubulares. Já a detecção precoce das doenças crônicas, antes do início da azotemia, permite uma intervenção que estabilize a função renal ou retarde o progresso da lesão ${ }^{2}$. Portanto, outros testes laboratoriais como a urinálise são importantes na precocidade da detecção de lesão renal, com a dosagem de proteinúria, 
microalbuminúria e determinação da relação proteína / creatinina urinária ${ }^{2,3,4}$.

A cintilografia fornece informaçóes quanto à funçáo renal global e individual pelo cálculo da taxa de filtraçáo glomerular e fluxo plasmático renal efetivo, além de avaliar a taxa de excreção renal. Porém, oferece poucos dados sobre a morfologia dos rins. Sabe-se que a ultrassonografia é um exame capaz de promover importante informação morfológica renal (de maneira mais apurada que o exame cintilográfico), e é um método de diagnóstico já bem estabelecido na rotina clínica de pequenos animais. Porém, rins morfologicamente comprometidos podem não ter sua função alterada e vice-versa ${ }^{5}$. Já com relação ao exame radiográfico, a urografia excretora pode avaliar de maneira qualitativa (portanto, subjetiva) a função individual dos rins; entretanto, a cintilografia oferece informaçóes quantitativas, ou seja, por meio de valores numéricos é capaz de determinar a taxa de filtração glomerular e a taxa de excreção renal de cada rim, individualmente ${ }^{6}$.

Apesar de o início da sua utilização na Medicina Veterinária datar a partir de 1970, a cintilografia (ou estudos por imagem na Medicina Nuclear) possui limitaçóes quanto à sua utilizaçáo, uma vez que o uso de material radioativo é controlado e o custo do equipamento é relativamente elevado e tornase restrito aos grandes centros de estudo. $\mathrm{O}$ radioisótopo mais utilizado para aplicaçóes na medicina veterinária é o ${ }^{99 m}$ Tecnécio $\left({ }^{99 \mathrm{~m} T c)}\right.$ devido às suas características físicas ${ }^{7}$. $\mathrm{O}$ radiofármaco é uma molécula marcada com um radioisótopo e administrada em pacientes humanos e animais. A molécula escolhida que será marcada pelo radioisótopo determina a maneira pela qual o radiofármaco vai se comportar química e biologicamente (local de instalação, trajeto, excreção). $\mathrm{Na}$ cintilografia renal, os rins são os órgãos-alvo, assim denominados por receberem a maior dose de radiaçáo do radiofármaco utilizado. O DTPA (ácido dietiltriaminopentoacético), um dos radiofármacos mais comumente utilizados na cintilografia renal, pode ser utilizado para determinar a taxa de filtraçáo glomerular $(\mathrm{TFG})^{7,8,9}$ e taxa de excreção renal ${ }^{10}$.

A cintilografia renal possui algumas vantagens e desvantagens na avaliação de rins de pequenos animais. Apesar de fornecer poucas informaçóes morfológicas, ser radioativo e sua disponibilidade ser limitada (devido ao alto custo do material e equipamento e as burocracias relacionadas ao uso de radioisótopos) $)^{6}$, este método ainda representa uma modalidade de diagnóstico por imagem importante, já que é simples, não estressante e náo invasiva ${ }^{7,11,12,13}$; é capaz de determinar a função renal global e individual $l^{6,8,11,14,15}$; avalia as afecçóes renais subclínicas ${ }^{8,9,16,17}$, os efeitos dos agentes nefrotóxicos ${ }^{9,16,18,19}$, o sucesso do transplante renal ${ }^{16,20,21}$; monitora a obstrução renal ${ }^{22,23}$, e não é prejudicada pela presença de líquido, ingesta, pela falta de gordura e pela desidratação ${ }^{6}$.

A diferenciação entre nefropatias obstrutivas e não obstrutivas constitui uma vantagem relevante do uso da cintilografia para avaliação renal, uma vez que a pielectasia observada ao exame ultrassonográfico não pode ser assim diferenciada nesta modalidade diagnóstica, e pode estar presente após fluidoterapia ou nas pielonefrites ${ }^{22,23}$. Assim sendo, sabendo que os felinos domésticos são comumente acometidos por doenças urinárias obstrutivas, a cintilografia pode fornecer informaçóes importantes nos quadros obstrutivos nesta espécie.

$\mathrm{Na}$ escassez de dados consistentes na literatura no que se refere à avaliação da excreção renal de felinos domésticos por meio de exames de cintilografia, o presente estudo objetiva verificar valores que determinam o tempo de excreção renal de animais clinicamente sadios, com a utilização de exames cintilográficos; verificação esta efetuada a partir da constatação de que os rins destes animais apresentam-se dentro dos limites da normalidade morfológica e funcional nos exames de radiografia simples, ultrassonografia, urografia excretora e análises laboratoriais rotineiras. 


\section{Materiais e Métodos}

Neste estudo experimental foram utilizados quinze gatos domésticos, sendo nove machos e seis fêmeas, adultos, os quais foram examinados clinicamente e amostras de sangue colhidas para a dosagem sérica de ureia e creatinina. Os animais foram considerados sadios mediante ausência de sinais clínicos sistêmicos e/ou específicos de afecçóes renais e normalidade da dosagem de ureia e creatinina. Previamente à realização dos exames cintilográficos, os animais foram submetidos a exames ultrassonográficos abdominais (aparelho $\mathrm{GE}^{\oplus} 200$ e GE $\mathrm{GE}^{\oplus}$ Logic 100 , com transdutores lineares de 7,5 MHz), exames radiográficos simples e urografia excretora. Foram incluídos no estudo os animais que não apresentaram alteraçóes morfológicas ao exame ultrassonográfico e radiográfico simples e animais que, qualitativamente, demonstraram ter função renal normal à urografia excretora.

As cintilografias foram realizadas no Setor Experimental do Serviço de Medicina Nuclear do Instituto de Radiologia do Hospital das Clínicas da Faculdade de Medicina da Universidade de São Paulo (SMN-HC-FMUSP), utilizando o radiofármaco ${ }^{99 \mathrm{~m}} \mathrm{Tc}$-DTPA, produzido na própria Instituição (gama-câmara modelo Orbiter ZLC 750, marca Siemens ${ }^{\oplus}$ ).

Os animais foram distribuídos em dois grupos, sendo o grupo 1 composto por dez animais náo anestesiados e o grupo 2, por nove animais anestesiados (sendo que quatro felinos foram submetidos aos exames por duas vezes - com e sem contenção química).

Os animais foram posicionados em decúbito ventral com contenção física apenas. $\mathrm{O}$ detector da gama-câmara foi posicionado dorsalmente à região epigástrica e $74 \mathrm{MBq}(2 \mathrm{mCi})$ do radiofármaco ${ }^{99 \mathrm{~m} T \mathrm{~T}-}$ DTPA foi injetado via intravenosa, com a utilização de um escalpe, na forma de injeção em "bolus", seguido de lavagem com solução salina. Após reposicionamento dos animais, o sistema de aquisição de imagens foi acionado e foram adquiridas 60 imagens com duração de 30 segundos de aquisição para cada uma, perfazendo um total de 30 minutos para cada exame. Pacientes que tiveram movimentação antes de completar os trinta minutos de exames pré-estabelecidos foram incluídos com o número de imagens disponibilizadas. Completado o exame, as imagens foram processadas utilizando o programa Image $J^{\oplus}$, com uma regiáa de interesse (ROI) sendo delimitada manualmente, com o auxílio do "mouse", ao redor de cada rim, numa imagem no qual estes pudessem ser inteiramente visibilizados (Figura 1). Por meio deste traçado, quantificou-se a radiação (contagem) emitida pelos rins em cada uma das 60 imagens, ou no número de imagens disponibilizadas antes da movimentação do animal (Figura 2). Assim, construiu-se um gráfico de contagem radioativa por tempo - o renograma - para a avaliação da excreção do radiofármaco de cada rim individualmente (Figura 3). Determinou-se, para cada animal, o tempo em que a radioatividade na região de interesse atingiu seu acúmulo máximo (chamado "T máximo"). As curvas foram extrapoladas para 3 minutos após esse acúmulo máximo, realizando-se uma regressão destes renogramas. Então, foi calculado o tempo necessário para essa radioatividade ser reduzida pela metade (chamado "T meio"). Os animais do grupo 2 foram submetidos à mesma técnica, posicionados da mesma maneira (Figura 4), com a introdução da anestesia geral como forma de contenção, utilizando zolazepam e tiletamina, via intramuscular, no volume de 1 $\mathrm{ml} / \mathrm{kg}$. Quatro animais utilizados nesta etapa já haviam sido avaliados por cintilografia, no processo de contenção física. Completado o exame, cada animal foi acondicionado em gaiolas específicas até a total eliminação do radiofármaco pela urina.

Para fins de avaliação e análise dos exames cintilográficos, os animais foram divididos em três grupos, a considerar: grupo 1, composto por dez animais nãoanestesiados, sendo oito machos e duas fêmeas; grupo 2, composto por nove animais anestesiados, sendo cinco machos e quatro fêmeas e grupo 3, composto pelo total de animais examinados (quinze), 


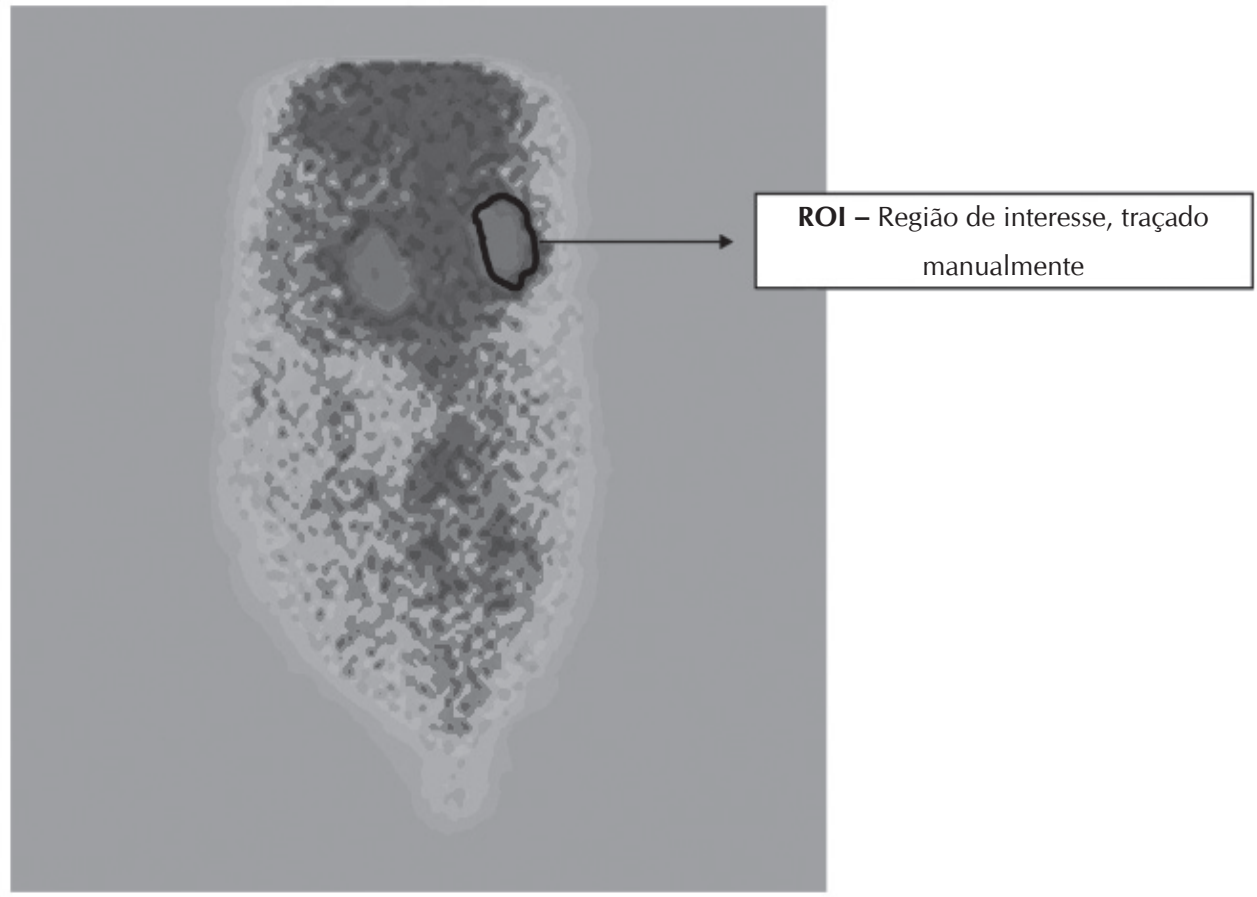

Figura 1- Imagem de cintilografia renal de felino doméstico, a partir da qual a região de interesse (ROI) é traçada ao redor de cada rim

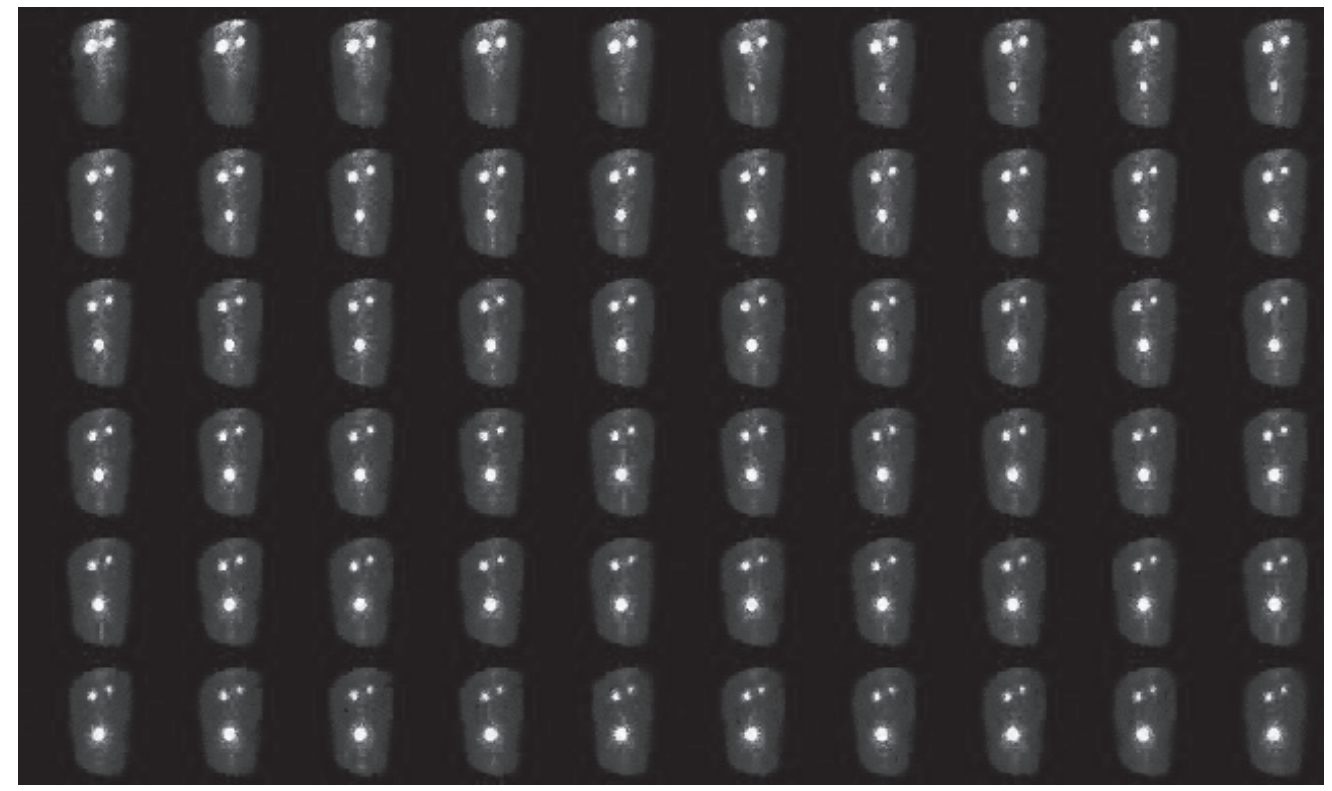

Figura 2- Sequência de imagens de cintilografia renal do felino fêmea do Grupo 2 (anestesiado) 
Animal anestesiado

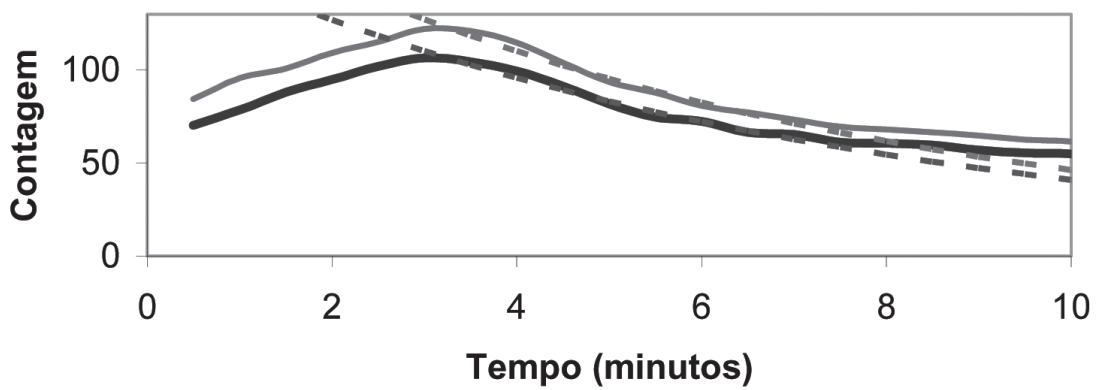

Rim Direito $\quad$ -

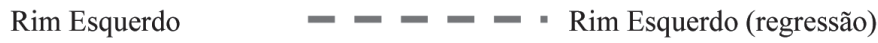

Figura 3- Curva de contagem de radioatividade renal por tempo (Renograma), obtida com o exame cintilográfico de felino doméstico anestesiado

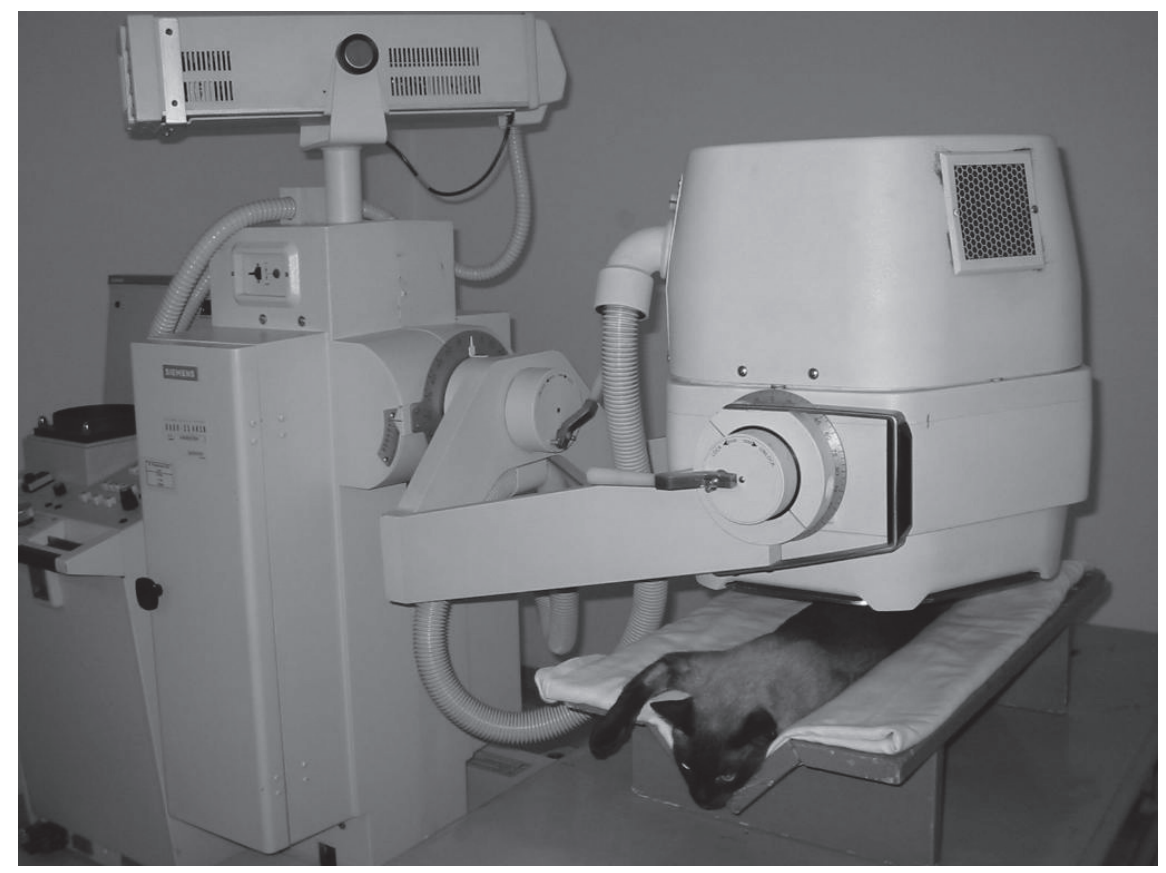

Figura 4- Aparelho de cintilografia (gama-câmara), com felino doméstico sendo submetido ao exame cintilográfico 
sendo nove machos e seis fêmeas. Foram realizadas análises estatísticas dos valores cintilográficos renais obtidos, considerando-se o "T máximo" e o "T meio" de cada um dos rins (direito e esquerdo), em cada um dos três grupos, considerando os machos e as fêmeas de cada grupo. Após a realização desta análise, os valores foram tratados pelo teste de "Mann-Whitney", com significância de $0,5 \%$ para verificação das diferenças estatísticas das amostras, comparando-se animais não-anestesiados e anestesiados, rins direitos e rins esquerdos e machos e fêmeas.

\section{Resultados}

Foram realizados ao total dezenove exames cintilográficos renais (de um total de 15 animais, quatro destes foram submetidos ao exame por duas vezes - com e sem a contenção química).

Os resultados obtidos para cada um dos rins, com relação ao T máximo (tempo que o radiofármaco levou para atingir sua concentração máxima na área de interesse) e T meio (tempo necessário para essa concentraçáo ser reduzida pela metade) em minutos, para os animais não-anestesiados e anestesiados, estão representados nas tabelas 1 e 2 ,

Tabela 1- Valores de T máximo e T meio (minutos) referentes aos rins esquerdo e direito dos felinos domésticos do Grupo 1 (animais não-anestesiados) - São Paulo - 2005

\begin{tabular}{|c|c|c|c|c|c|c|}
\hline \multirow[b]{2}{*}{ A } & \multirow[b]{2}{*}{ Sexo } & \multicolumn{2}{|c|}{$\operatorname{Rim} E$} & \multicolumn{2}{|c|}{$\operatorname{Rim} D$} & \multirow{2}{*}{$\begin{array}{c}\text { Imagens } \\
\text { obtidas }\end{array}$} \\
\hline & & T máx. & T $1 / 2$ & T máx. & T 1/2 & \\
\hline 1 & $M$ & 3,5 & 10,18 & 4,0 & 7,22 & 60 \\
\hline 2 & $M$ & 1,5 & 3,36 & 1,5 & 3,12 & 17 \\
\hline 3 & M & 2,0 & 5,5 & 2,0 & 5,75 & 13 \\
\hline 4 & M & 3,0 & 6,57 & 3,5 & 8,73 & 27 \\
\hline 5 & $M$ & 2,5 & 6,85 & 2,5 & 7,23 & 37 \\
\hline 6 & $M$ & 3,5 & 10,09 & 3,5 & 9,59 & 60 \\
\hline 8 & $M$ & 3,0 & 6,42 & 2,5 & 6,73 & 47 \\
\hline 9 & $\mathrm{~F}$ & 1,5 & 7,94 & 1,5 & 6,61 & 47 \\
\hline 10 & $\mathrm{~F}$ & 1,5 & 3,01 & 1,5 & 1,03 & 60 \\
\hline 11 & M & 3,0 & 8,47 & 3,0 & 8,12 & 17 \\
\hline
\end{tabular}

Tabela 2- Valores de T máximo e T meio (minutos) referentes aos rins esquerdo e direito dos felinos domésticos do Grupo 2 (animais anestesiados) - São Paulo - 2005

\begin{tabular}{|c|c|c|c|c|c|c|}
\hline \multirow[b]{2}{*}{ A } & \multirow[b]{2}{*}{ Sexo } & \multicolumn{2}{|c|}{$\operatorname{Rim} E$} & \multicolumn{2}{|c|}{$\operatorname{Rim} D$} & \multirow{2}{*}{$\begin{array}{c}\text { Imagens } \\
\text { obtidas }\end{array}$} \\
\hline & & T máx. & T $1 / 2$ & T máx. & $\mathrm{T}_{1 / 2}$ & \\
\hline 1 & $M$ & 4,0 & 5,29 & 3,5 & 4,73 & 60 \\
\hline 2 & $M$ & 3,0 & 5,24 & 3,0 & 5,35 & 60 \\
\hline 3 & $M$ & 2,0 & 4,92 & 2,5 & 4,63 & 60 \\
\hline 4 & $M$ & 4,0 & 5,66 & 4,0 & 6,9 & 60 \\
\hline 7 & $\mathrm{~F}$ & 2,5 & 3,73 & 2,5 & 3,84 & 60 \\
\hline 12 & $\mathrm{~F}$ & 3,5 & 5,15 & 3,0 & 6,46 & 60 \\
\hline 13 & $\mathrm{~F}$ & 3,0 & 4,8 & 3,0 & 4,91 & 60 \\
\hline 14 & $\mathrm{~F}$ & 2,5 & 4,97 & 3,0 & 7,82 & 60 \\
\hline 15 & M & 3,0 & 5,45 & 3,0 & 8,91 & 60 \\
\hline
\end{tabular}


respectivamente, assim como o número de imagens obtidas para cada um dos animais.

Considerando os animais nãoanestesiados, obteve-se uma média de $2,55 \pm 0,92$ minutos para o $\mathrm{T}$ máximo em rins direitos e 2,5 $\pm 0,81$ minutos em rins esquerdos. Em relação ao T meio, a média foi de $6,41 \pm 2,58$ minutos para rins direitos e 6,83 $\pm 2,45$ minutos para rins esquerdos.

Já em relação aos animais anestesiados, obteve-se uma média de 3,05 $\pm 0,46$ minutos para o $\mathrm{T}$ máximo em rins direitos e 3,05 $\pm 0,68$ minutos em rins esquerdos. Em relação ao T meio, a média foi $5,95 \pm 1,76$ minutos para rins direitos e 5,02 $\pm 0,55$ minutos para rins esquerdos.

Pode-se perceber, por meio do teste de Mann-Whitney, que não houve diferença estatisticamente significante entre os valores obtidos de rins esquerdos e direitos, nem entre animais anestesiados e náo anestesiados, assim como também não houve diferença entre machos e fêmeas.

\section{Discussão}

O exame cintilográfico renal é um método de diagnóstico por imagem que apresenta a grande vantagem de oferecer informação da função do órgão individualmente; porém, há uma menor resolução espacial e, consequentemente, uma avaliação morfológica inferior quando comparada a outras técnicas, como a ultrassonografia. Sabe-se que a avaliação morfológica náo traduz a função renal, pois rins morfologicamente normais podem ter sua função comprometida e rins com sua arquitetura alterada podem ser funcionalmente normais ${ }^{5}$. Assim, a ultrassonografia renal, metodologia que avalia de maneira apurada a morfologia deste órgão, deve ser adotada como um método de diagnóstico adicional na avaliação de rins de pequenos animais. A urografia excretora avalia a função renal individual, porém de maneira qualitativa, pois não oferece valores numéricos que traduzam a taxa de filtração glomerular e a taxa de excreção renal, como faz o exame cintilográfico. Portanto, apesar da urografia excretora ser um método rápido, pouco dispendioso e não possuir limitaçóes quanto ao material e equipamento necessários como a cintilografia, este método radiográfico contrastado faz a avaliação funcional dos rins apenas subjetivamente. ${ }^{6}$

O foco principal de nosso estudo foi a verificação de valores que representam a excreção renal mediante estudos cintilográficos de perfusão renal. $\mathrm{O}$ trabalho foi focado na determinação do tempo necessário para o radiofármaco atingir metade da sua concentração nos rins, a partir do tempo de acúmulo máximo.

Inicialmente, estabelecemos que os animais náo sofreriam qualquer processo de sedação ou anestesia, para se evitar qualquer alteração dos valores procurados decorrente do uso de drogas. Desta maneira, os animais foram posicionados em decúbito ventral, com os membros torácicos e pélvicos tracionados cranial e caudalmente, respectivamente, e o detector da gama-câmara foi posicionado dorsalmente à porçáo cranial do abdome. Este posicionamento permite que o detector esteja o mais próximo possível do órgão de interesse, aumentando a estatística de contagem e a resolução espacial do sistema, permitindo que os órgáos possam ser visibilizados o mais próximo possível do seu tamanho real. Em outros estudos ${ }^{11,14,24}$, o detector da gama-câmara também estava localizado dorsalmente à porção cranial do abdome, porém os animais foram posicionados lateralmente, o que náo altera a contagem, uma vez que a espessura do tecido atravessado pela radiação é a mesma. $\mathrm{O}$ estabelecimento do posicionamento do animal em nosso estudo foi semelhante ao protocolo utilizado em experimentos com coelhos, no Setor de Medicina Nuclear do Hospital das Clínicas da Faculdade de Medicina da Universidade de São Paulo. Neste protocolo, o posicionamento dos animais em decúbito ventral é facilitado pela utilização de uma calha, mantendo o animal estável e viabilizando a manutenção da posição do paciente sem auxílio manual.

$\mathrm{O}$ processo de aquisição de imagem também seguiu o protocolo rotineiramente 
utilizado na mesma instituição, ou seja, administração de 37 a $74 \mathrm{MBq}$ (1 a 2 mCi) do radiofármaco e aquisiçấo de imagens dinâmicas a 30 segundos por quadro, num período de 30 minutos, obtendo-se, então, um total de 60 imagens. Como observado em literatura, a aquisição das imagens podem ser adquiridas de inúmeras maneiras, variando de uma imagem por segundo ${ }^{25}$ a uma imagem a cada 15 segundos ${ }^{11}$. Também se encontra na literatura grande variaçáo com relação ao tempo de duração do exame. As imagens podem ser adquiridas durante 1 minuto (fase vascular do exame) ) $^{9,25}$ até 30 minutos $^{22,23}$, variando de acordo com o tipo de avaliação a ser realizada (taxa de filtração glomerular ou taxa de excreção). No presente estudo, foi avaliada a taxa de excreçâo renal; portanto, é necessário um maior tempo (30 minutos) para que seja possível a obtenção dos valores requeridos para tal avaliação.

O renograma, ou a curva construída com as imagens a partir do momento da injeçáo do radiofármaco até o término do exame, pode ser dividido em três partes. A primeira representa a chegada do radiofármaco na área de interesse (aporte sanguíneo), com um aumento rápido da radioatividade no órgão; a segunda representa o tempo em que a radioatividade atinge sua máxima contagem dentro da área de interesse (fase de acúmulo); e a terceira fase é definida como aquela onde o radiofármaco começa a deixar o órgão e representa a fase de excreção ${ }^{10}$. A fase inicial, ou vascular, definida como as primeiras imagens durante o primeiro minuto, não foi por nós avaliada, uma vez que o interesse do estudo foi verificar os valores de $\mathrm{T}$ máximo e T meio para a avaliação da excreção renal e esses valores se encontram numa fase mais avançada do renograma. Portanto, a fase vascular não foi de interesse no presente estudo e o fato de náo ter sido avaliada não interferiu nos resultados.

A não utilização da anestesia mostrou-se inadequada, uma vez que, de dez animais avaliados, apenas três permitiram a conclusão do tempo total do estudo, isto é, mantiveram-se imóveis pelos 30 minutos.
A associação de agentes empregada é bastante utilizada em felinos domésticos; é considerada segura, com período hábil suficiente para a realizaçáo do procedimento. Os resultados demonstraram que não houve variação estatística nos tempos de acúmulo máximo e no tempo de excreção renal do radiofármaco quando do uso ou náo deste agente anestésico. Portanto, este pode ser utilizado nessa modalidade diagnóstica sem preocupações em relação às possíveis alterações causadas por ele na excreção renal, diferentemente daqueles outros protocolos cuja interferência parece ser maior ${ }^{10,15,26}$.

Não há grande disponibilidade no Brasil de centros de Medicina Nuclear que realizam exames cintilográficos em pequenos animais, pois a metodologia requer equipamento especializado e local apropriado, com legislação rigorosa devido ao material radioativo utilizado na técnica. Porém, apesar dessa dificuldade, sugere-se o aumento da demanda da realização deste exame, uma vez que o mesmo é possível de ser realizado no país e possui grandes vantagens, como: avaliar as afecçóes renais subclínicas ${ }^{8,9,16,17}$; avaliar os efeitos dos agentes nefrotóxicos ${ }^{9,16,18,19}$, o sucesso do transplante renal ${ }^{14,16,21}$; monitorar a obstrução renal ${ }^{22,23}$ e principalmente determinar a função renal individual, fundamental na avaliação do rim contra-lateral na decisão de uma nefrotomia ou nefrectomia diante de cálculos renais e/ou hidronefroses ${ }^{14,17}$.

\section{Conclusões}

Com base nos resultados obtidos e nas circunstâncias metodológicas no qual foi proposto esse estudo, acredita-se que é passível a realizaçáo da cintilografia renal sem maiores consequências em gatos domésticos, sendo que a técnica deve ser preferencialmente realizada em animais anestesiados, permitindo-se dessa forma uma avaliação mais apurada da funçáo quantitativa renal. Considera-se que a cintilografia renal pode ser utilizada na avaliaçáo da taxa de excreção renal em gatos domésticos, sendo sua grande vantagem a avaliaçáo quantitativa 
renal de maneira individual, devendo a técnica ser incorporada aos outros métodos de diagnóstico por imagem que analisam a morfologia e a função renal qualitativa, como ultrassonografia e urografia excretora, respectivamente.

\section{Agradecimentos}

À CAPES e ao Serviço de Medicina Nuclear - Instituto de Radiologia do Hospital das Clínicas da Universidade de São Paulo.

\title{
Urinary excretion rate assessment by scintigraphy in domestic cats
}

\begin{abstract}
In internal medicine of domestic cats, some imaging diagnosis modalities, such as ultrasonography, radiography and intravenous pylogram are spreadly used. Scintigraphy is a non-invasive technique, which provides functional information of individual kidneys; however, it is regarded not ordinary nowadays. The aim of this study was to verify the time of excretion of each particular kidney in domestic cats by scintigraphy, and these animals were presented with normal ultrasonographic and radiographic parameters. We used 15 cats, nine males and six females, and they were divided into awake and anesthetized cats. We calculated the time the radiopharmaceutical takes to reach the maximum activity in the kidneys (T max.) and the time it takes to decrease into half of this value (half-time). There was no difference between the awake and anesthetized cats, nor between the right and left kidneys, and no difference between male and female either.
\end{abstract}

\section{Referências}

1 OSBORNE, C. A.; FINCO, D. R. Canine and feline nephrology and urology. Media: Williams \& Wilkins, 1995.

2 GRAUER, G. F. Early detection of renal damage and disease in dogs and cats. Veterinary Clinics of North America: Small Animal Practice, v. 35, n. 3, p. 581596, 2005.

3 LEES, G. E. Early diagnosis of renal disease and renal failure. Veterinary Clinics of North America: Small Animal Practice, v. 34, n. 4, p. 867-885, 2004.

4 GRAUER, G. F. Measurement, interpretation, and implications of proteinuria and albuminuria. Veterinary Clinics of North America: Small Animal Practice, v. 37, n. 2, p. 283-295, 2007.

5 WALTER, P. A.; FEENEY, D. A.; JOHNSTON, G. R.; O'LEARY, T. P. Ultrasonographic evaluation of renal parenchymal diseases in dogs: 32 cases (1981-1986). Journal of American Veterinary Medical Association, v. 191, n. 8 , p. 999-1007, 1987

6 RIVERS, B. J.; JOHNSTON, G. R. Diagnostic imaging strategies in small animal nephrology. Veterinary
Keywords: Nuclear Medicine. Scintigraphy. Kidneys. Feline.
Clinics of North America: Small Animal Practice, v. 26, n. 6, p. 1505-1517, 1996.

7 TWARDOCK, A. R.; KRAWIEC, D. R.; LAMB, C. R. Kidney scintigraphy. Seminars in veterinary Medicine and Surgery, v. 6, n. 2, p. 164-169, 1991.

8 DANIEL, G. B.; MITCHELL, S. K.; MAWBY, D. SACKMAN, J. E.; SCHMIDT, D. Renal nuclear medicine: a review. Veterinary Radiology \& Ultrasound, v. 40, n. 6, p. 572-587, 1999.

9 LORA-MICHIELS, M.; ANZOLA, K.; AMAYA, G.; SOLANO, M. Quantitative and qualitative scintigraphic measurements of renal function in dogs exposed to toxic doses of gentamicin. Veterinary Radiology \& Ultrasound, v. 42, n. 6, p. 553-561, 2001.

10 LOURENS, D. C.; DORMEHL, I.; GOOSEN, D. J. The feasibility of a renogram study in dogs with radiopharmaceutical ${ }^{99 m}$ TC-DTPA. Journal of the South African Veterinary Association, v. 53, n. 4, p. 243248, 1982.

11 KRAWIEC, D. R.; BADERTSCHER, R. R.; TWARDOCK. A. R.; RUBIN, S. I.; GELBERG, H. B. Evaluation of ${ }^{99 m}$ Tc-diethylenetriaminepentacetic acid nuclear imaging for quantitative determination of the glomerular filtration rate of dogs. American 
Journal of Veterinary Research, v. 47, n. 10, p. 2175-2179, 1986.

12 ITKIN, R. J.; KRAWIEC, D. R.; TWARDOCK. A. R.; GELBERG, H. B. Quantitative renal scintigraphic determination of effective renal plasma flow in dogs with normal and abnormal renal function, using ${ }^{99 m}$ Tc-mercaptoacetyltriglycine. American Journal of Veterinary Research, v. 55, n. 12, p. 1660-1665, 1994.

13 ITKIN, R. J.; KRAWIEC, D. R.; TWARDOCK. A. R.; GELBERG, H. B.; KORITZ, G. D. Evaluation of the single-injection plasma disappearance of technetium$99 \mathrm{~m}$ mercaptoacetyltriglycine method for determination of effective renal plasma flow in dogs with normal or abnormal renal function. American Journal of Veterinary Research, v. 55, n. 12, p. 1652-1659, 1994.

14 KRAWIEC, D. R.; TWARDOCK. A. R.; BADERTSCHER, R. R.; DANIEL, G. B.; DUGAN, S J. Use of ${ }^{99 \mathrm{~m} T c-d i e t h y l e n e t r i a m i n e p e n t a c e t i c ~ a c i d ~ f o r ~}$ assessment of renal function in dogs with suspected renal disease. Journal of American Veterinary Medical Association, v. 192, n. 8, p. 1077-1080, 1988.

15 NEWELL, S. M.; KO, J. C.; GINN, P. E.; HEATONJONES, T. G.; HYATT, D. A.; CARDWELL, A. L.; MAURAGIS, D. F.; HARRISON, J. M. Effects of three sedative protocols on glomerular filtration rate in clinically normal dogs. American Journal of Veterinary Research, v. 58, n. 5, p. 446-450, 1997.

16 BALOGH, L.; ANDÓCS, G.; THURÓCZY, J.; NÉMETH, T.; LÁNG, J.; BODÓI, K.; JÁNOKI, G. A. Veterinary nuclear medicine: scintigraphy examinations - a review. Acta Veterinaria Brno, v. 68, p. 231-239, 1999.

17 GOOKIN, J. L.; STONE, E. L.; SPAULDING, K. A. BERRY, C. R. Unilateral nephrectomy in dogs with renal disease: 30 cases (1985-1994). Journal of American Veterinary Medical Association, v. 208, n. 12, p. 2020-2026, 1996.

18 MARSHALL, K. L.; CRAIG, L. E.; JONES, M. P.; DANIEL, G. B. Quantitative renal scintigraphy in domestic pigeons (Columba livia domestica) exposed to toxic doses of gentamicin. American Journal of Veterinary Research, v. 64, n. 4, p. 453-461, 2003.

19 NEUWIRTH, L.; KUPERUS, J. H.; CALDERWOODMAYS, M.; GASKIN, J. Comparative study of Indium-111 leuckocytes and nephrosonography for detection of experimental pyelonephritis in dogs. Veterinary Radiology \& Ultrasound, v. 36, n. 3, p. 253-258, 1995.

20 HALLING, K. B.; GRAHAM, J. P.; NEWELL, S. P.; ELLISON, G. W.; DETRISAC, C. J.; MARTIN, F. G.; VANGILDER, J. M.; GROSSMAN, D. Sonographic and scintigraphy evaluation of acute renal allograft rejection in cats. Veterinary Radiology \& Ultrasound, v. 44, n. 6, p. 707-713, 2003.

21 NEWELL, S. M.; ELLISON, G. W.; GRAHAM, J. P.; GINN, P. E.; LANZ, O. I.; HARRISON, J. M.; SMITH, J. S.; VAN GILDER, J. M. Scintigraphy, sonography, and histologic evaluation of renal autotransplantation in cats. American Journal of Veterinary Research, v. 60, n. 6, p. $775-779,1999$

22 BARTHEZ, P. Y.; SMEAK, D. D.; WISNER, E. R.; DUFFEY, M.; CHEW, D. J.; DiBARTOLA, S. P. Effect of partial ureteral obstruction on results of renal scintigraphy in dogs. American Journal of Veterinary Research, v. 60, n. 11, p. 1383-1389, 1999.

23 BARTHEZ, P. Y.; SMEAK, D. D.; WISNER, E. R.; DiBARTOLA, S. P.; CHEW, D. J. Ureteral obstruction after ureteroneocystostomy in dogs assessed by technetium ${ }^{99 \mathrm{~m}} \mathrm{Tc}$ diethylenetriamine pentaacetic acid (DTPA) scintigraphy. Veterinary Surgery, v. 29, n. 6, p. 499-506, 2000

24 URIBE, D.; KRAWIEC, D. R.; TWARDOCK. A. R.; GELBERG, H. B. Quantitative renal scintigraphy determination of the glomerular filtration rate in cats with normal and abnormal kidney function, using ${ }^{99 \mathrm{~m} T \mathrm{C}-}$ diethylenetriaminepentacetic acid. American Journal of Veterinary Research, v. 53, n. 7, p. 1101-1107, 1992.

25 KAMPA, N.; WENNSTROM, U.; LORD, P.; TWARDOCK, R. T.; MARIPUU, E.; EKSELL, P.; FREDRIKSSON, S. Effects of region of interest selection and uptake measurement on glomerular filtration rate measured by ${ }^{99 \mathrm{~m}} \mathrm{Tc}$-DTPA scintigraphy in dogs. Veterinary Radiology \& Ultrasound, v. 43, n. 4, p. 383-391, 2002.

26 GRIMM, J. B.; GRIMM, K. A.; KNELLER, S. K.; TRANQUILLI, W. J.; CROCHIK, S. S.; BISCHOFF, M. G.; PODOLSKI, J. L. The effect of a combination of medetomidine-butorphanol and medetomidine, butorphanol, atropine on glomerular filtration rate in dogs. Veterinary Radiology \& Ultrasound, v. 42, n. 5, p. 458-462, 2001. 